\title{
The effect of Doppler correction on measures of storm track intensity
}

\begin{abstract}
In climate research, the intensity of a storm track is often measured by the band pass filtered eddy kinetic energy, geopotential variance or related variables. The interpretation of such fields disregards the influence the advection speed might have on these measures. In this paper the need for a Doppler corrected storm track measure is discussed. A Doppler corrected measure is defined and applied to 10 years of ECMWF reanalysis data, correcting the storm track measure for spatial and temporal variability of the advection speed. The storm track intensity is also calculated correcting only for the temporal variability of the advection speed. It is also related to fluctuations of the NAO teleconnection pattern. The Doppler correction suggests that (1) maximum and minimum baroclinic activity is found somewhat downstream of the locations indicated by non-corrected measures, (2) the storm track activity estimated by conventional measures is much too low in the areas of the eastern ends of the storm tracks, (3) the monthly mean time series of the strength of a storm track, as estimated by conventional measures, is strongly influenced by the variability of the advection speed at times, (4) the strength of the storm track seems to be less strongly connected with teleconnection patterns such as NAO or with the background mean flow speed than usually thought on the basis of conventional Eulerian statistics.
\end{abstract}

\footnotetext{
U. Burkhardt $\cdot$ I. N. James

Department of Meteorology, University of Reading, Reading, UK

Present address: U. Burkhardt $(\bowtie)$

Institut für Physik der Atmosphäre, DLR Oberpfaffenhofen, 82234 Wessling, Germany

E-mail: ulrike.burkhardt@dlr.de

Tel.: + 49-8153-282561

Fax: + 49-8153-281841
}

\section{Introduction}

The strength and low frequency variability of a storm track are often determined by calculating the variance of band pass filtered variables in the upper troposphere. Quantities such as geopotential variance or velocity variance in the upper troposphere, filtered with a band pass filter with cut-offs at periods of 2 and 6 days are commonly analysed. Such measures are intended to pick out the regions where baroclinic disturbances move or develop (Blackmon 1976; Blackmon et al. 1977; Lau 1988; Wallace et al. 1988). In the northern hemisphere, such measures emphasize the two storm tracks localized over the ocean basins. These apparently start in the vicinity of the stationary troughs over the eastern continents and terminate near the stationary ridges over the western coasts of the continents (Lau 1988). Storm track localization may be connected with the life cycles of baroclinic eddies (Simmons and Hoskins 1978) and possibly with the barotropic modulation of the evolving disturbances by the large scale flow (Lee 1995; Swanson et al. 1997).

The strength, location and extent of the storm track are very dependent on the large scale flow (see, for example, Mullen 1986; Lau 1988; Metz 1989). The storm tracks in turn force the large scale flow (Hoskins et al. 1983). In particular, storm track variability as deduced from band pass filtered variances and covariances is strongly related to the variability of the teleconnection patterns and to the strength of the zonal background flow in general (Lau 1988). The low frequency variability of the storm track measures has a strong influence on the climate experienced on the continents (Fraedrich et al. 1993) and so has been studied widely for the observed climate (Nakamura 1989; Lau 1988), in paleoclimates (Kageyama et al. 1999; Dong and Valdes 1998) and in climate change studies (e.g. Hall et al. 1994; Ulbrich and Christoph 1999). The low frequency variability of the strength of the storm track is analyzed using the same band pass filtered variance measure. A period of low 
variance is then interpreted as a period of low baroclinic activity.

A storm track measure based on a fixed frequency band relies on the ability to discriminate between baroclinic and large scale eddies on the basis of the phase speed $\omega_{g} k^{-1}$ of the disturbances relative to the ground, approximately in the zonal direction, where $k$ is the zonal wavenumber. But the phase speed of the disturbances relative to the ground depends upon the background flow speed, $u$, as well as on the intrinsic phase speed $\omega_{i} k^{-1}$ of the disturbance relative to the moving atmosphere:

$\omega_{g} k^{-1}=\omega_{i} k^{-1}+u(x, t)$.

If the intrinsic phase speed of the disturbance was independent of the background flow, which would be the case if the variations of $u^{\prime}$ were barotropic in structure, there would be an obvious problem with using band pass filtered statistics as a measure of the intensity of a storm track. The variability of the flow would determine which disturbances were included in the storm track measure. If the flow were strong, lower frequency $\omega_{i}$ intrinsic variability would be included in the measure than if the flow were weaker. In this situation, a Doppler correction of the storm track measure, which eliminated the influence of the large scale flow would be necessary. On the other hand, if the zonal wavenumber $k$ of the disturbance changed as the strength of the background flow changed, then the westward phase speed of the disturbance relative to the flow would change, resulting in a different eastward phase speed relative to the ground. In the extreme case, the phase speed would change by just the right amount to keep the frequency relative to the ground constant. Were that the case, the usual kind of storm track measure would include a set of disturbances with characteristics independent of the background flow speed and a Doppler correction would not be necessary.

The aim of this paper is to determine whether a Doppler correction is appropriate and to determine whether the storm track measure would be significantly affected by Doppler correction. A method for implementing a Doppler correction is introduced and the implications of Doppler correction for the structure and variability of the storm track are examined. Section 2 describes the data used in this study and illuminates the problem further. The choice of the large scale wind speed $u(x, t)$ (Eq. 1) is discussed in Appendix 1. The relationship between the background flow speed and the zonal wavenumber of a disturbance is further examined in theory, Appendix 2, and in observed data, Sect. 3.

The results presented in Sect. 3 suggest that the wavenumber of baroclinic disturbances is neither exactly independent of the background flow speed, nor that the related change in intrinsic frequency exactly compensates for the variability of the flow speed. Thus, the usual storm track measures not only reflect the variability of the baroclinic activity but also, at least to some degree, the variability of the background flow. Therefore a
Doppler corrected storm track measure has potential to provide valuable additional information about the strength of the storm track. The corrected and uncorrected measures provide two extreme estimates of the strength of the storm track. In the remainder of this paper, the intrinsic phase speed of the disturbances will be assumed to be independent of the background flow speed.

Section 4 describes our method used to Doppler correcting a storm track measure, involving extended empirical orthogonal function analysis (EEOF-analysis) or multichannel singular spectrum analysis (MSSA). The storm track measure is corrected, in Sect. 5 for the spatial and temporal variability of the background flow, and in Sect. 6 for the temporal variability of the background flow speed only. In Sect. 5, the effect of Doppler correction on the location of the storm track, on the location of the maximum and minimum of storm track intensity and on the relation of the intensity in the storm track and in the gap area is analysed. Section 6 considers the month to month variability of the storm track intensity and whether it changes if the storm track measure is corrected for only the temporal fluctuations of the large scale flow speed. The Doppler correction may be especially extensive at times of extremes in the teleconnection indices. This might lead to an overestimation of the connection between storm track activity and the strength of the teleconnection pattern.

\section{Data}

Data for the northern hemisphere winter seasons, defined as December, January and February, from 1983/84 to $1992 / 93$, taken from the ECMWF initialized reanalysis data (Gibson et al. 1997) were analysed. The meridional wind at $30 \mathrm{kPa}$ and the absolute wind speed at $70 \mathrm{kPa}$ were given every $12 \mathrm{~h}$ on a $2.8^{\circ}$ latitude-longitude grid. The data were averaged over the latitudes from 35 to $66^{\circ} \mathrm{N}$. Data were not weighted according to their latitude. In the following sections, the latitudes over which the data were averaged varied slightly with the mean position of the storm track: thus, at the eastern end of the storm tracks the latitudinal belt was slightly further to the north than at the western end of the stormtrack.

An introduction to the problems associated with defining a storm track using a temporal band-pass filter results from visualizing the data in form of a Hovmoeller plot (Fig. 1a). The meridional wind at $30 \mathrm{kPa}$ shows variability with a variety of length scales and frequencies. There are mountain waves, quasi-stationary waves and high frequency waves. The latter are most pronounced in the storm track areas over the ocean.

A Hovmoeller diagram of the 2-6 day band pass filtered meridional wind (Fig. 1b) shows the high frequency disturbances. They are most pronounced in the storm track areas over the oceans. However, the disturbances are often remarkably long lived and often 

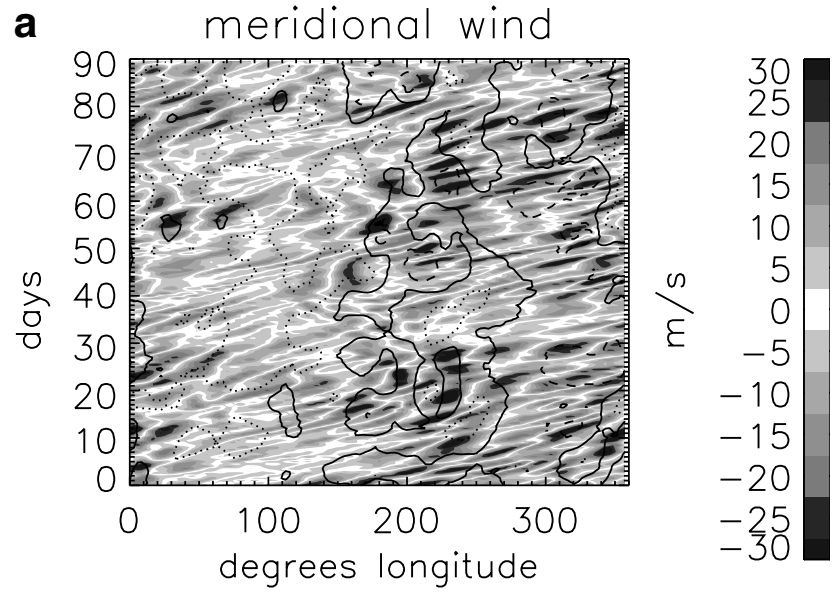

b
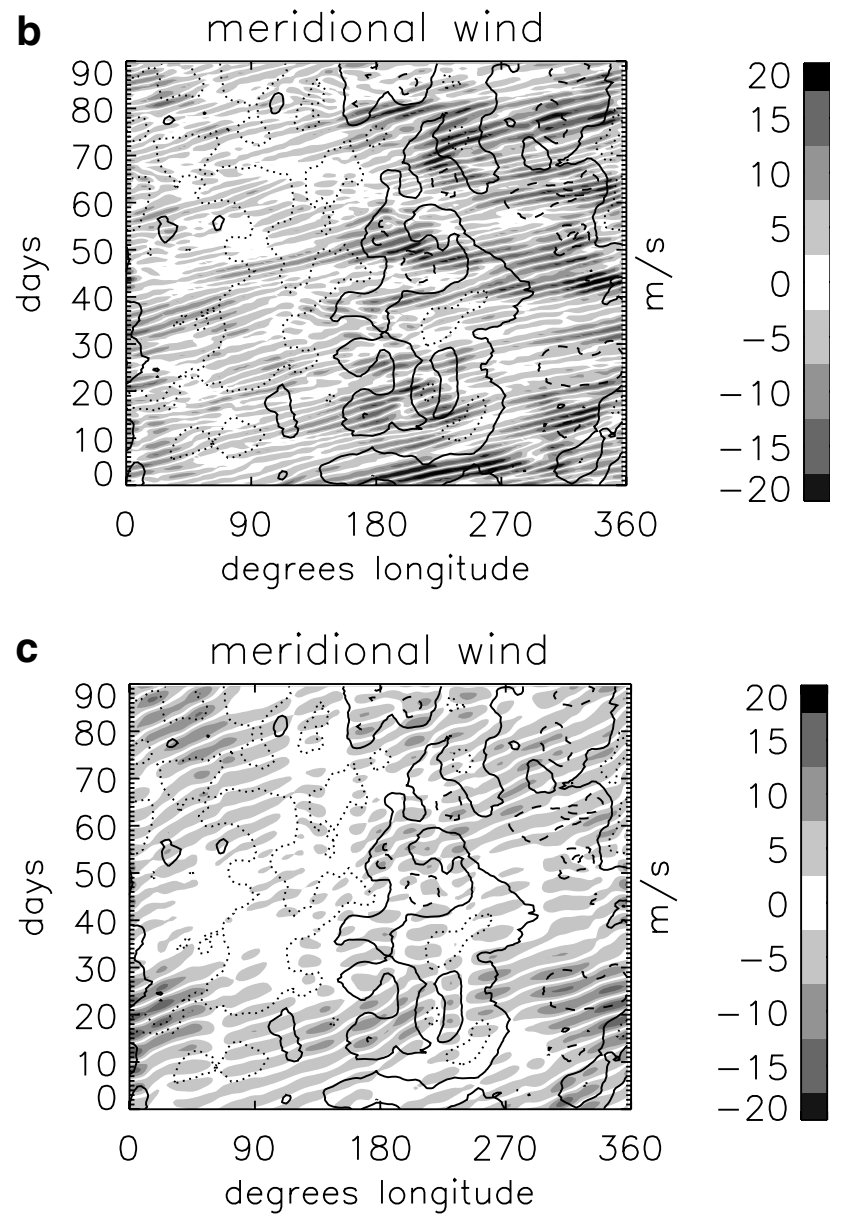

Fig. 1 Hovmoeller diagram of the a unfiltered, b 2-6 day band pass filtered and $\mathbf{c} 6-10$ day band pass filtered meridional wind anomaly at $30 \mathrm{kPa}(\mathrm{DJF} 90 / 91)$ averaged over the latitudes from 35 to $66^{\circ} \mathrm{N}$. Isolines indicate the 10 day low pass filtered absolute wind speed at $70 \mathrm{kPa}$ with contours being drawn at $10 \mathrm{~m} \mathrm{~s}^{-1}$ (dotted), $14 \mathrm{~m} \mathrm{~s}^{-1}$ (solid) and $18 \mathrm{~m} \mathrm{~s}^{-1}$ (dashed)

propagate right over North America. The intensity of the disturbances is quite variable and can decrease quite suddenly. This might be due to a number of reasons. First, a disturbance might decay. Secondly, the phase speed of the disturbance relative to the ground might change, which could mean that the disturbance is no longer included in the band pass range of frequencies. In that case the disturbance will show up in an adjacent frequency band. Such a change in phase speed may be accompanied by a decrease in the large scale wind speed at $70 \mathrm{kPa}$ which is also shown in Fig. 1b. In that case, as long as the wavelength is unchanged, the decrease of variability in the filter band would be due to the Doppler effect, and should not be interpreted as a decrease in storm track activity. The validity of Doppler correction using the $70 \mathrm{kPa}$ large scale flow is discussed in Appendix 1 where we show that the variability of the large scale low pass filtered $70 \mathrm{kPa}$ flow is a good approximation to the barotropic flow variability.

Figure 1c shows the 6-10 day band pass filtered meridional wind velocity at $30 \mathrm{kPa}$. Sometimes the variability of the meridional wind in this filter band resembles the variability in the 2-6 day band. In fact, at times a strong meridional wind speed anomaly can be seen at the same time and location as in the 2-6 day band, indicating that substantial variability is associated with disturbances whose periods are close to the 6 day cut off. If at the same time, the large scale flow happens to be slower than usual, then there would be a prima facie case for applying a Doppler correction. If, on the other hand, the large scale wind speed is larger than usual it would appear that more of the variability should be excluded from the storm track measure.

For climatological studies, it may not be very important if one particular cyclone were included in the storm track or not. But it may be important to know if there are systematic errors when calculating storm track activity. Such systematic errors would not be eliminated by a general widening of the filter band. That would simply shift the problem to a different frequency band. Since there is no gap in the spectrum, there is no ideal cut off frequency which would circumvent this problem. Choosing a larger frequency band (e.g. 2-8 days) results in the same patterns as obtained by a $2-6$ day filter but with about $20 \%$ more variance (Trenberth 1981, 1991). Such systematic error is likely to be largest when comparing the storm track intensity at locations where the mean wind speed is significantly different. A systematic error may also appear when determining the temporal variability of the storm track. For example, Doppler correction could affect the strength of the storm track in different phases of teleconnection patterns such as the NAO (Wallace and Gutzler 1981), since the NAO is associated with variability of the strength of the midlatitude westerly flow.

\section{Dependence of the wavenumber on the large scale flow}

A Doppler corrected measure can be defined according to Eq. 1. If the wavelength of the disturbance and the background wind speed are known then $u(x, t) k$ can be split into $\bar{u}(x, t) k+u^{\prime}(x, t) k$ and the term due to the temporal (or temporal and spatial) variability of the flow 
can be subtracted from the estimated frequency relative to the ground. The Doppler corrected measure is accurate if the scale of the disturbance is not affected by the background wind speed. The uncorrected measure is accurate if the change in the background flow is exactly compensated by a change in the intrinsic phase speed of the disturbance. Such a compensation can arise from, for example, a change in the scale of the disturbance. As detailed in Appendix 2 in the case of $u^{\prime}$ being a barotropic flow anomaly, $k$ does not change with $u^{\prime}$. In cases when $u^{\prime}$ is correlated with the vertical wind shear, idealised baroclinic instability theory does not give a clear prediction which if either of the two cases is more appropriate. The Eady model predicts that the scale of the most unstable disturbance will be independent of the flow. The Charney model predicts that the wavelength of the most unstable wave will depend on the vertical shear of the flow and therefore on the flow if $u^{\prime}$ is correlated with the vertical wind shear. This is likely to be a small effect since our large scale low frequency $u^{\prime}$ in $70 \mathrm{kPa}$ is a good approximation of the anomaly of the barotropic flow (Appendix 1). However, it is undesirable to restrict the analysis to the baroclinic growth stage of the lifecycles, which normal mode theory describes. WKB theory (Gill 1982) also predicts the wavelengths of small amplitude waves to depend on the mean flow but the assumptions made are very idealized (Appendix 2) and apply neither to the growth or decay phase. Whether barotropic effects play an important part in modulating storm track activity has not been established so far (Appendix 2). In the following we shall consider the relevance of a Doppler corrected storm track measure to the whole life cycle of baroclinic disturbances, including both the growth and decay phases. This section presents an analysis of the dependence of the wavelength on the large scale wind speed in observational data over the Atlantic.

The wavelengths of atmospheric disturbances were estimated from the unfiltered and band pass filtered meridional wind velocity. The analysis was carried out in the region of the Atlantic storm track between longitudes 280 and $360^{\circ} \mathrm{E}$ in order to exclude strong influences of orographic waves or of other low frequency variability. The meridional wind speed at $30 \mathrm{kPa}$ in the area of the Atlantic storm track was analyzed for ten consecutive winter seasons (DJF) using the data described in Sect. 2. The meridional wind field was searched for local maxima and minima and the half wavelength of a cyclone was approximated by the distance between an adjacent minimum and maximum. Consequently, even small and weak cyclones were analyzed, and not only the cyclones with the largest transport properties. Then the low pass filtered wind speed at $70 \mathrm{kPa}$ was averaged over the area defined by the half wavelength of the disturbance. This low pass filtered wind speed was regarded as an estimate of the background wind speed. The wavelengths were then plotted versus the low pass filtered wind speed. Wavelengths at low wind speeds are mainly connected with disturbances in the eastern part of the analysed area but may also be sometimes connected with disturbances at the American jet exit at times of low wind speed. An increase of the wavelength with increasing background flow speed that is equal in both the unfiltered and filtered data set would point to an exact compensation of the variability of the background flow speed by a change in intrinsic phase speed of the disturbances. In that case Doppler correction would not be justified. If, on the other hand, the wavelength does not increase with increasing background flow in the unfiltered data, or increases much less than in the filtered data, then Doppler correction of the storm track measure would be necessary.

Figure $2 \mathrm{a}$ shows the number of cyclones found for each background wind speed and wavelength combination in the unfiltered and filtered data set. The wavelengths range from about 8 grid points $\left(1.6 \times 10^{6} \mathrm{~m}\right)$ to 26 grid points $\left(5.2 \times 10^{6} \mathrm{~m}\right)$. The crosses represent the medians of the estimated wavelengths with associated large scale wind speeds lying in a bin of $1.0 \mathrm{~m} \mathrm{~s}^{-1}$ width. A least squares regression line was fitted to the medians, rather than the points themselves. This was done because the points are not expected to lie on a line but rather to lie within a band of values. This band of values is determined in the unfiltered data by the range of
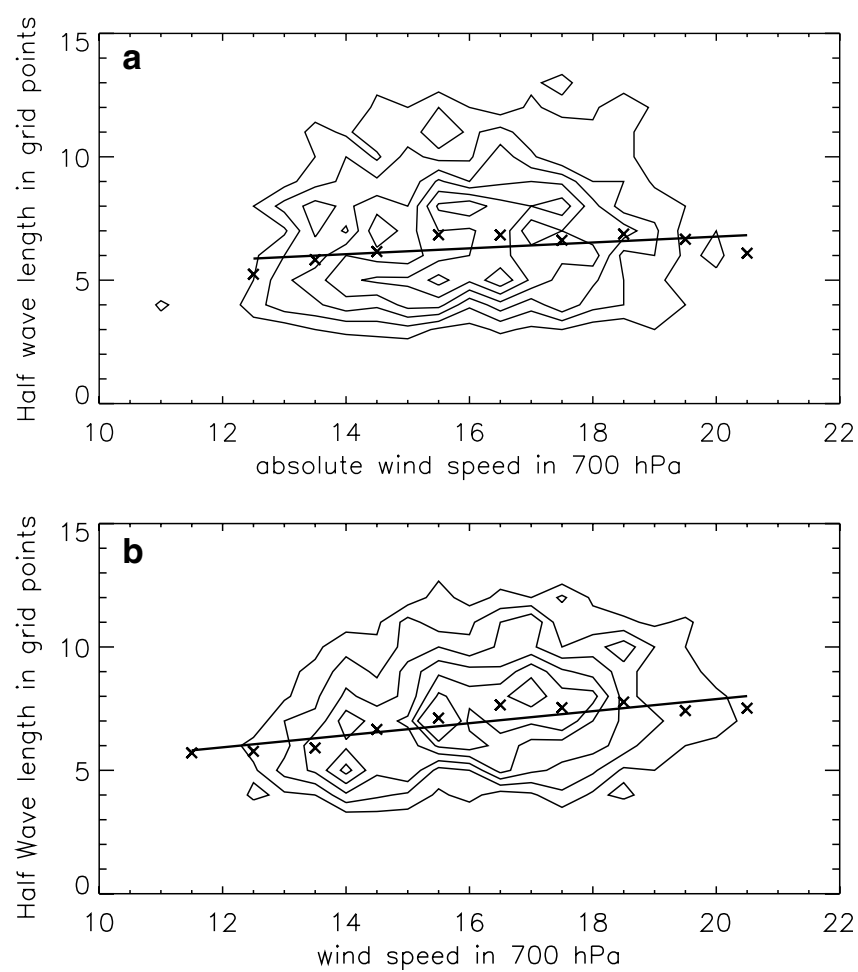

Fig. 2 Number of disturbances with a certain half wavelength as estimated from the $\mathbf{a}$ unfiltered and $\mathbf{b}$ filtered meridional wind field at $30 \mathrm{kPa}$ and the 10 day low pass filtered flow speed at $70 \mathrm{kPa}$ in the area of the Atlantic storm track. Isolines have a contour interval of 5. Crosses indicate the median of the wavelength at a certain strength of the flow and the line indicates the least squares fit to the medians 
baroclinic disturbances found over the Atlantic and in the filtered data set by the filter band. The slope of the regression line is 0.12 with a standard error of 0.06 . The variation of the wavelength with flow speed is not statistically significant (even when choosing a significance level of $90 \%$, see Wilks 1995). The least-squares regression line is very close to the sample mean so that the variance of the regression line divided by the total variance (coefficient of determination) of the medians is only 0.33 . Nevertheless the data suggest that there may be a slight dependency. If instead of the unfiltered meridional wind, the 2-6 day bandpass filtered meridional wind is used (Fig. 2b) then the medians of the wavelengths show a significant trend $(0.24$ with error 0.04 ) with the large scale wind speed, even if a rejection level of $1 \%$ is chosen. The variance described by the regression line relative to the total variance (coefficient of determination) is 0.8 . If the regression line is calculated by weighting the medians and the errors with the population in the bins, the slopes, of 0.09 and 0.29 for the unfiltered and filtered data set, respectively, are significantly different.

Overall the data indicate that the wavelength is slightly dependent on the zonal wind speed. This change in wavelength does not change the intrinsic phase speed of the disturbances in such a way as to ensure that after filtering the same disturbances are still included in the data set as before filtering. Although we have only analyzed the Atlantic storm track, we expect that the same would hold for the Pacific. As a result, at times or places with high background wind speed, disturbances with longer wavelengths and slower eastward movement are included in the filter band than at times or places where the flow is weak. The variability of the large scale wind speed almost certainly influences our picture of the location and intensity of the storm tracks when using variance measures. But how the picture would change if an appropriate correction of the storm track measure for fluctuations of the background wind were included is not obvious.

\section{Methods}

To apply a Doppler correction, the data set needs to be decomposed into different wave components, each with a zonal and temporal scale. Extended empirical orthogonal function (EEOF) analysis provides a means of performing this decomposition. The frequency of each EEOF pattern can be Doppler corrected using its associated wavenumber and a suitably filtered background wind.

A temporal filter was constructed based on an EEOFanalysis as follows. The meridional wind velocity was filtered with a 10 day high-pass Lanczos filter (Duchon 1979, later referred to as the pre-filter) in order to eliminate the very low-frequency oscillations, and was averaged over latitudes between 35 and $66^{\circ} \mathrm{N}$. The field was then decomposed into the main oscillatory patterns using an EEOF analysis (Weare and Nasstrom 1982), also referred to as MSSA analysis (Plaut and Vautard 1993).

In a second step, the frequency and length scale of the oscillatory patterns was estimated. Using those space-time EOFs, a time series of the band-pass filtered meridional wind anomaly was constructed. The bandpass filter may be defined with and without a Doppler correction. The band-pass filtered time series was composed of the variability described by those space-time patterns for which the estimated or corrected periods are lower than 6 days.

\subsection{Extended empirical orthogonal function analysis}

Extended empirical orthogonal function analysis determines those space-time patterns in a data set which describe the maximum variance of the data. This means that the first pattern describes a maximum amount of variance and the succeeding patterns describe a maximum amount of variance in the remaining part under the condition that their time series are uncorrelated.

The EEOF expansion is defined as follows. The state vector of a data set $X(l, t)$ at time $t$ is $[X(1, t+1), X(2$, $t+1), \ldots \ldots ., X(L, t+1), X(1, t+2), X(2, t+2), \ldots . X(L$, $t+2), \ldots \ldots X(1, t+W), X(2, t+W), \ldots . X(L, t+W)]$, with a coordinate for the longitude $1 \leq l \leq L$ and $L$ the number of space coordinates. The number of time steps included in the state vector is denoted $W$ and is called the window length. The expansion of the state vector in the so called space-time EOFs is defined here as

$X(l, t+j)=\sum_{k=1}^{L W} a_{k}(t) E_{k}(l, j) \quad 1 \leq l \leq L ; 1 \leq j \leq W$,

with the pattern $E_{k}$ having a longitude and time coordinate, $l$ and $j$, respectively. This expansion looks very similar to a normal expansion of a field in EOFs. Indeed, in the case where $W=1$, the expansion is a conventional EOF-expansion. In the case where the number of space coordinates $L=1$, the expansion is a singular spectrum analysis (SSA)-expansion. The space-time EOFs are determined as eigenvectors from a covariance matrix of dimension $L W$ which includes the spatial and temporal covariance structure of the data set. The amplitude of the $k$ th pattern, $a_{k}$, is also called the spacetime principal component.

Here the reconstruction of the field is not uniquely defined since both the patterns and the amplitudes include a time dependency. In this paper, the reconstruction using the $k$ th space-time-EOF at longitude $l$ and time step $t$ is defined as:

$$
X_{k}(l, t)=\frac{1}{W} \sum_{j=1}^{W} a_{k}(t-j) E_{k}(l, j) \quad W \leq t \leq N-W+1,
$$


with $N$ being the length of the time series. Accordingly, reconstructions for $t \leq W$ and $t \geq N-W-1$ can be defined which consist of fewer terms (Plaut and Vautard 1993). By summing over the first few space-time-EOFs, the original data field $X$ can be approximated.

In this study the EEOF analysis was made in $60^{\circ}$ longitude sections, thereby including a whole wavelength of a wavenumber 6 disturbance. The window length was chosen to be 10 days (20 time steps) in order to be able to resolve the longest periodicities inherent in the prefiltered data. Choosing a much lower cut off frequency of the prefilter and a correspondingly longer window length would have meant that the high frequency variability could not be resolved and the temporal localization of a spell of oscillation would have been low (see Sect. 4.3).

As noted earlier, EEOF-analysis was used in this study as a means of decomposing the data set into different wave components with a particular zonal scale and an associated temporal scale. EEOF-analysis was not used to determine statistically significant periodicities inherent in the data set. Even though the parameter choices were kept constant, different space-time EOFs were obtained in different seasons or areas since the EEOFs adapt to the data.

\subsection{Extended empirical orthogonal function patterns and reconstruction}

When a data set is decomposed into space-time patterns, wave-like features in the data set are described by pairs of patterns which both describe a similar amount of variance, given by the eigenvalues. The first pairs of patterns in particular are in approximate phase quadrature.

The 10 day high pass filtered meridional wind field over Europe for the winter season 1990/91 and its reconstruction using just the first 12 EEOFs are shown in Fig. 3. Using only the first few EEOFs for reconstruction, the low frequency and large spatial scale structure of the field is reconstructed. The reconstruction using 12 EEOFs looks like a smoothed version of the original 10 day high pass filtered field and describes about $65 \%$ of the variability of the original field (with pattern 11 and 12 describing between 3 and $6 \%$ ). Variability that does not
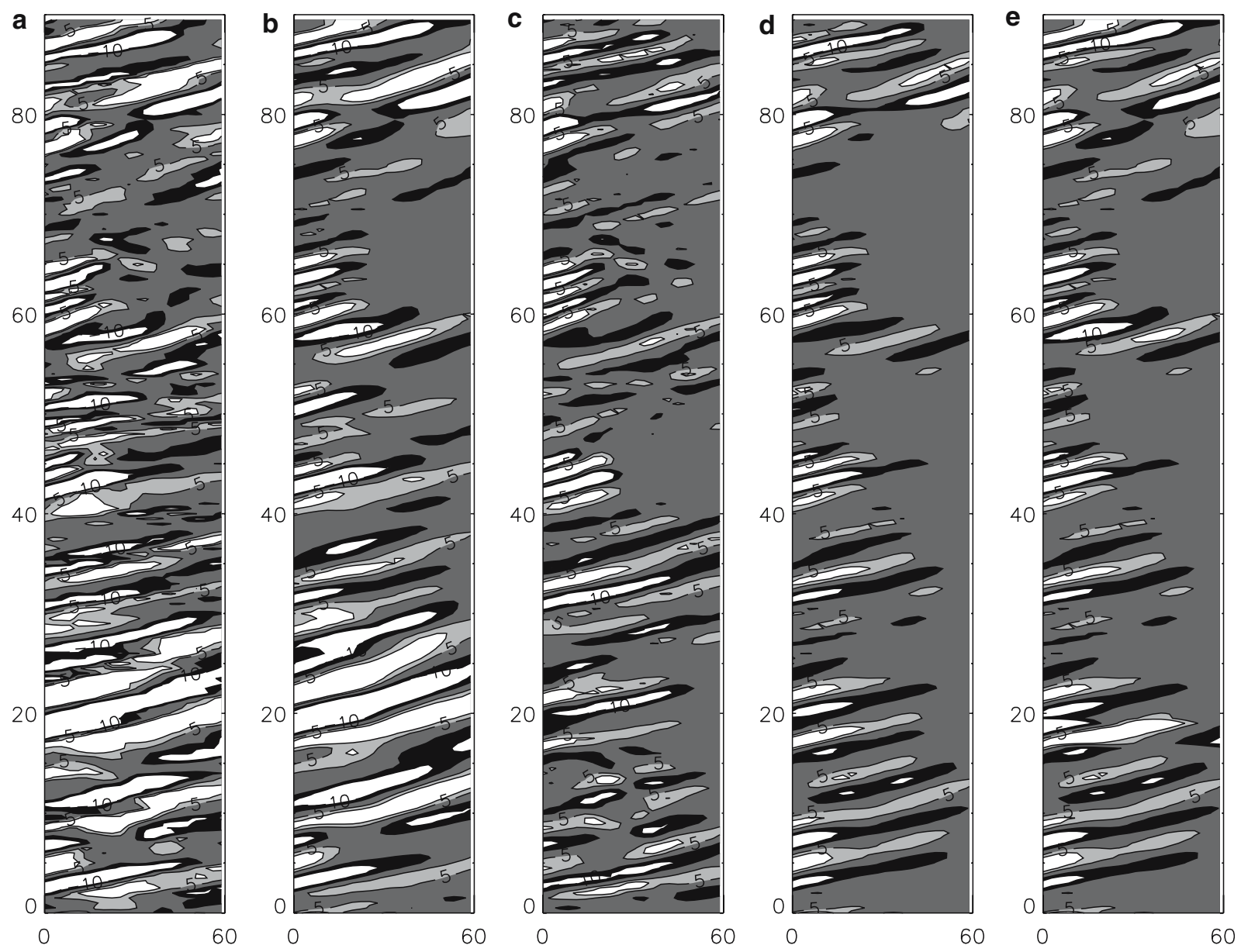

Fig. 3 Time series (DJF 90/91) of the a Lanczos filtered and b with 12 space-time patterns reconstructed 10 day high-pass filtered meridional wind component between $0^{\circ}$ and $60^{\circ} \mathrm{E}$ and the time series of the 2-6 day band-pass filtered meridional wind component c using a Lanczos filter, d using a EEOF filter, e Doppler corrected EEOF filter when correcting towards a time and area averaged background wind speed. Different shadings are used for values exceeding $-10,-5,5$ and $10 \mathrm{~m} \mathrm{~s}^{-1}$ 
appear to be associated with propagating disturbances (e.g. between days 60 and 70 in the eastern part of the domain) are not reproduced, while variability that propagates extremely fast (e.g. around day 50) are reproduced in a smoothed form using the first 12 EEOFs.

From the EEOF patterns, the zonal scale $k$ and the frequency $\omega_{g}$ were estimated, using the autocorrelation function. The time lagged autocorrelation of a pattern was computed and averaged over the spatial coordinates to obtain $\omega_{g}$. Similarly, the space lagged autocorrelation of the pattern was computed and averaged over the time steps to obtain $k$. For example if a pattern describes a sinusoidal wave then the space or time lag at which the autocorrelation function equals -1 is half a wavelength or half a waveperiod. Reliable estimates of $\omega_{g}$ and $k$ can therefore be obtained only if the waves are fairly regular in frequency and wavelength. The higher EEOFs were very irregular and so only the first 12 EEOFs were used for the reconstruction.

\subsection{Extended empirical orthogonal function filter}

A bandpass filter can be constructed using only those of the first 12 EEOFs whose estimated $\omega_{g}$ lies between prescribed cut off frequencies. The main difference between using EEOF-analysis rather than classical spectral methods for filtering is that the basis functions in EEOFanalysis are local in time and are determined adaptively, whereas classical spectral methods use prescribed sine and cosine functions. Furthermore using EEOF-analysis, the amplitude of the patterns can vary in time, allowing this method to deal with intermittent information. The larger the window length, the sharper is the spectral resolution and the poorer is the temporal localization of any intermittent spell of oscillation. If the window length is longer than the spell of oscillation, then the amplitude of the oscillation will be underestimated within the spell and overestimated in its neighbourhood. In the limit when the window length tends to infinity, the eigenvectors tend to pairs of sines and cosines and the associated eigenvalues tend to the corresponding spectral density values (Vautard 1995). The choice of the window length is crucial and depends on the range of frequencies being considered. EEOF resolves periods in the range of $W / 5$ to $W$ (Plaut and Vautard 1993). Therefore it is necessary to prefilter the data if the window length is to be kept short. Choosing $W=10$ days means that variability with periods between 2 and 10 days can be resolved, covering the filter band of 2-6 days to a large extent even at times of relatively low wind speeds. It also means that intermittent spells of oscillation can be localized temporally quite well. A broader filter band which retained disturbances connected with lower frequency variability would have eliminated the high frequency end of the spectrum.

A filter obtained by rejecting EEOFs with frequencies outside a given band will be referred to as an
EEOF-filter. Although similar to a conventional Lanczos bandpass filter, it will not give identical results for a number of reasons:

1. Both filters deal very differently with intermittent information (see above).

2. The response function of an EEOF-filter and a Lanczos filter are different. The EEOF-filter damps variability with frequencies between the two cut off frequencies only slightly, provided they are associated with large zonal scales. In contrast to the Lanczos filter, it is able to distinguish between coloured noise and superimposed oscillations of the same frequency because of their different spatial structure. The Lanczos filter passes variability independently of the spatial scale and reduces the variability with frequencies equal to the cut off frequencies by one half. The 2-6 day Lanczos filter, used to compare the EEOF filtered data to, damps the variability by 0.75 at periods of 1.92 and 6.9 days and by 0.825 at periods of 1.87 and 7.6 days.

3. Since only the first few EEOFs are used for the reconstruction, only a portion of the overall variance is described. The percentage of variance of the 10 day high pass filtered field described by the first 12 EEOFs is $65 \%$. Particularly the variance connected with very small spatial and temporal scales is excluded. When constructing the 2-6 day band pass filtered field any patterns that have periods outside the filter band are excluded as well. Consequently, both the EEOF filtered fields look like low pass filtered versions of the associated Lanczos filtered fields. Nevertheless, synoptic scale disturbances are resolved well in the EEOF 2-6 day filtered field.

Using a fixed number of EEOFs for filtering is not problematic since it is always the first few EEOFs that are connected with the large spatial and temporal scales and get therefore rejected by a high pass filter. Therefore there is no variability in the rank of the patterns describing low frequency variability, which could have caused spurious low frequency variability of the variance passed by the filter.

Figure 3 compares the 2-6 day Lanczos filtered meridional wind field over Europe for the season 1990/ 91 (Fig. 3c) and the EEOF-filtered field (Fig. 3d). Generally, the two filtered fields look similar. The EEOF filtered field looks like a smoothed version of the Lanczos filtered field. Around day 33, the variability over the eastern part of the domain in the Lanczos filtered field (Fig. 3c) is connected with a frequency lower than the low frequency cut off and therefore excluded by the EEOF filter (Fig. 3d). Since the response function of the Lanczos filter goes only slowly to zero, some of this low frequency variability is still contained in the Lanczos storm track measure. Around day 50 and between days 60 and 70, the Lanczos filtered time series contains variability that does not seem to be connected with baroclinic disturbances; apparent phase speeds are 
partly westward. This variability is not reconstructed using the EEOF filter because it was not recognized as being connected with a wave like structure. In the 10 day high pass filtered field, this variability was already excluded from the reconstruction (see Sect. 4.2).

\subsection{The Doppler corrected measure}

A Doppler corrected filtered field can be constructed using the strength of the flow and the zonal scale of the disturbance. The Doppler correction is applied to the cut off frequencies of the filter, depending on the area averaged flow anomaly, $u^{\prime}(x, t)$, and on the zonal scale, $k$, of the wave pattern

$\omega_{\mathrm{co}, u 0}=\omega_{\mathrm{co}}-u^{\prime}(x, t) k$,

where $\omega_{\mathrm{co}}$ is the cutoff frequency of the uncorrected filter and $\omega_{\mathrm{co}, u 0}$ is the Doppler corrected cut off frequency. The flow anomaly $u^{\prime}$ was averaged over a large area so as not to correct the phase speed of the disturbances using their own flow field. The Doppler correction was carried out towards a globally and temporally constant background flow speed of $16 \mathrm{~m} \mathrm{~s}^{-1}$, a speed representative of the jet cores at $70 \mathrm{kPa}$ (Sect. 5) or towards the time and area mean wind speed, depending on the analysis area (Sect. 6). Then $u^{\prime}(x, t)$ is the time anomaly of the 10 day low-pass filtered $70 \mathrm{kPa}$ wind speed. If the frequency of a wave pattern lies within the Doppler corrected cut off frequencies, then that pattern will be included in the storm track measure. Because the wind fluctuates, $\omega_{\text {co } u 0}$ is calculated at each time step for each pattern independently. A particular pattern may therefore be included in the Doppler corrected storm track measure at some time steps and not at others. This does not mean that the Doppler corrected field would include large temporal discontinuities since the construction of the field includes a summing over 20 time steps of the EEOF amplitude multiplied with the EEOF itself (see Eq. 3). The Doppler correction is insensitive to the number of retained EEOFs since it is only the first few EEOFs with the longest time scales which account for the correction.

In Fig. 3e, the Doppler corrected filtered field over Europe for the season 1990/91 is shown, where an area averaged low-pass filtered wind speed was used to make the correction. Some of the differences between the Doppler corrected and uncorrected fields are due to variability being moved into the field that was not included in the uncorrected field (see days 70-75). But most of the time, disturbances are simply somewhat stronger in the corrected field than in the uncorrected field. Examples are at around day 20 and between days 55 and 60.

Figure 4 shows the corrected (solid) and uncorrected (dotted) storm track intensity over Europe $\left(0-60^{\circ} \mathrm{E}\right)$ in the winter $84 / 85$. The data were high-pass prefiltered with a 10 day cut off, and then EEOF filtered using a high pass filtering band of 2-6 days. The Doppler corrected measure was also recalculated using a prefilter of 12 days with the other parameters as before and with the 12 day prefilter and an additional adjustment in the filter band to 2-6.5 days. All three corrected measures agree very well in the absence of Doppler corrections. When the background wind anomaly is large, the Doppler corrected time series is strongly dependent on the exact frequency associated with the patterns. Therefore a season and location with extremely strong Doppler correction has been selected in order to demonstrate the possible sensitivity to these parameters. The three Doppler corrected time series all include much more of the variability around day 22 and 86 in the filter band. All three measures also exclude the variability around day 55. Around day 41 and 46 the situation is different. At these times, a change in the prefilter means that the estimated frequency of the pattern responsible for the Doppler correction changes slightly. As a result, no Doppler correction is made. Therefore at those times, the Doppler corrected measure is equal to the uncorrected measure. The change in the estimated frequency is relatively small. Hence the associated variability at those times is Doppler corrected into the frequency band if the band pass frequency range is extended up to 6.5 days.

\section{Correcting for temporal and spatial variability}

In this section, we address the question of whether Doppler correction could significantly change the mean
Fig. 4 Time series of the uncorrected (dotted line) and Doppler corrected meridional wind component. For the Doppler corrected time series a 10 day prefilter and a $2-6$ day filtering band (solid line), a 12 day prefilter and a 2-6 day filtering band (dashed line) or a 12 day prefilter and a 2-6.5 day filtering band (dash dotted line) have been used

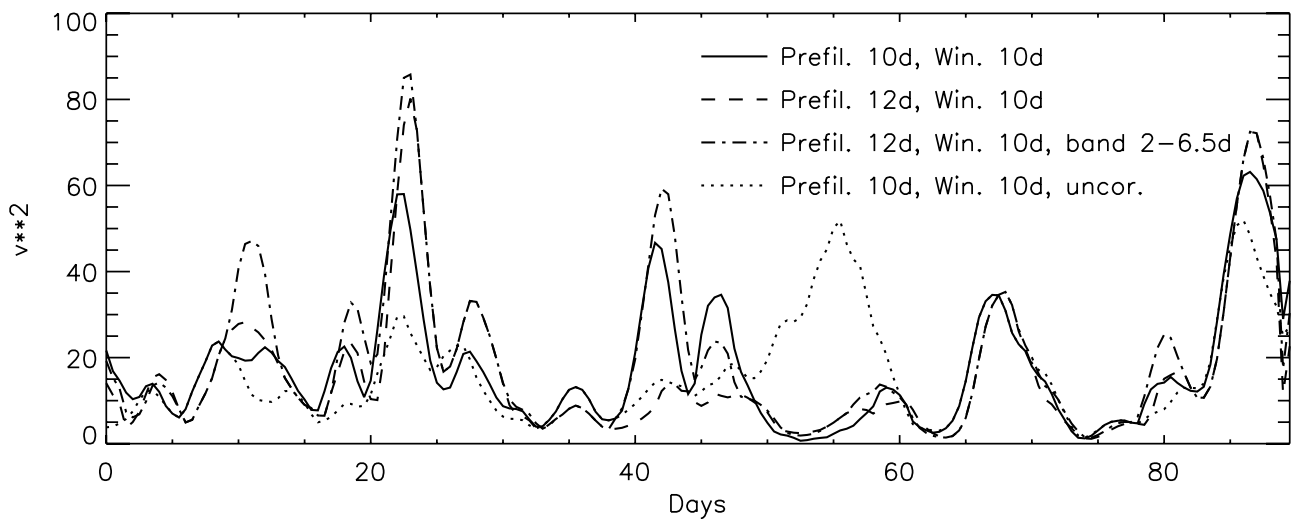


position, extent or strength of the observed storm track. The Lanczos filter, the EEOF-filter and the Doppler corrected filter were each applied to the ten winter seasons (DJF) of ERA data. The seasonal average and the monthly mean and daily time series of the variance of the meridional wind at $30 \mathrm{kPa}$ were calculated. For the correction, the anomaly of the large scale wind speed relative to a wind speed of $16 \mathrm{~m} \mathrm{~s}^{-1}$, a value typical for the jet cores at $70 \mathrm{kPa}$, has been used. This value ensures that little activity is corrected out of the 2-6 day period band in the vicinity of the jet cores. Even though the EEOF-analysis was applied in $60^{\circ}$ sections, the area averaged background wind speed and the storm track variability in an area was calculated in the inner $30^{\circ}$ in order to get a better longitudinal resolution. All the parameters of the prefilter (10 day high pass), window length (10 days) and number of retained EEOFs (12) were kept constant.

\subsection{The time mean storm track}

Figure 5 shows the variance of the band pass filtered meridional wind speed for different longitude sections, averaged over ten DJF seasons. Even though the response functions of the Lanczos and EEOF filter were different, they produced similar results, with closer agreement in the Pacific area than in the Atlantic area. When Doppler correcting towards a wind speed of $16 \mathrm{~m} \mathrm{~s}^{-1}$, large positive corrections were applied to the estimates of storm track intensity in the areas of the eastern Pacific, western America and Europe. In the western Atlantic area, the time mean background wind speed was above $16 \mathrm{~m} \mathrm{~s}^{-1}$ and, associated with that, a significant amount of variability was corrected out of the storm track band. If the storm track measure had been corrected towards a higher wind speed, less variance would have been corrected out of the storm track band

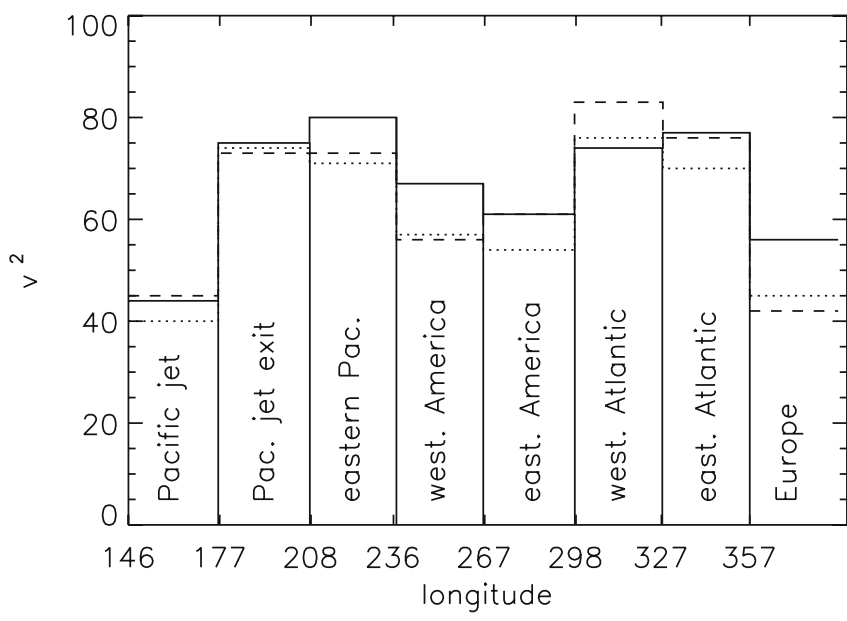

Fig. 5 Variance of the meridional wind anomaly in $30 \mathrm{kPa}$ (units $\mathrm{m}^{2} \mathrm{~s}^{-2}$ ) in different areas after application of a EEOF-filter (dashed), Lanczos filter (dotted) and after Doppler correction (continuous) over the western Atlantic and more variance into the storm track band in all other regions.

Generally for both the Pacific and Atlantic storm track, the area of Doppler-corrected storm track intensity was extended towards the east compared to the uncorrected storm track. The maximum storm track activity moved slightly further to the east. In both storm tracks, the variance over the eastern part of the ocean basins and the western coast of the continents increased strongly. This decreases the difference between the maximum storm track intensity and the intensity in the area that is usually referred to as the end of the storm track. The gap between the Pacific and Atlantic storm tracks itself narrows and shifts further to the east.

\subsection{Monthly variability}

The seasonal mean changes in the storm track intensity which can be ascribed to Doppler corrections are made up of strongly contrasting corrections in individual months. Figure 6 shows the monthly mean time series (ten DJF seasons) of the storm track activity, both uncorrected and Doppler-corrected, together with the associated background wind speed anomaly in the European area. The mean large scale wind speed was much lower than the wind speed towards which the scheme was correcting. Hence the correction was very strong at times. Over most of the time series, Doppler correction did not change the estimate of storm track activity very much. For example, the correction was very
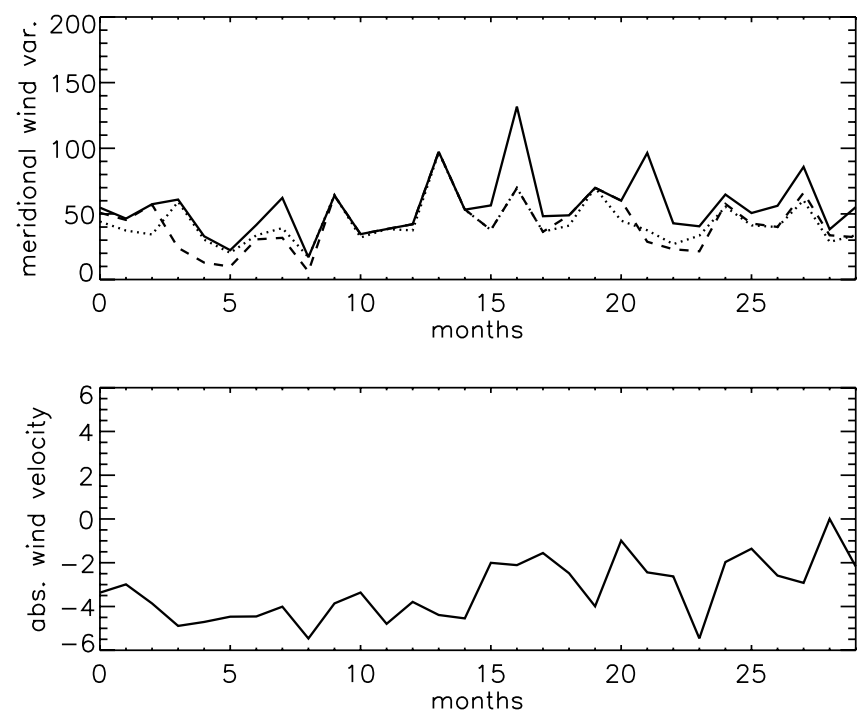

Fig. 6 Monthly mean time series of the meridional wind variance (in $\mathrm{m}^{2} \mathrm{~s}^{-2}$ ) over Europe. The dashed line indicates the uncorrected time series, the solid line the Doppler corrected time series when correcting towards a mean background wind speed of $16 \mathrm{~m} \mathrm{~s}^{-1}$, and the dotted line the corrected time series when correcting towards the time averaged wind speed in the area of interest (i.e. correct for temporal variability only). The lower plot shows the deviation of the background wind velocity at $70 \mathrm{kPa}$ from $16 \mathrm{~m} \mathrm{~s}^{-1}$ (units $\mathrm{m} \mathrm{s}^{-1}$ ) 
small or zero in the seasons $86 / 87$ and $87 / 88$ (months 9 14). In other months, for example, January 1989 (month 16) and December 1990 (month 21), Doppler correction doubled the estimate of storm track activity and changed a month associated with low storm track activity into a month associated with high activity. Thus, a Doppler-corrected measure would identify different months as very active months when compared to an uncorrected measure.

In the eastern Atlantic area (Fig. 7) the anomaly of the large scale wind speed was much smaller and therefore the Doppler correction was generally much smaller than in the European area. Some months exhibit Doppler-correction of variance out of the storm track filter band and some months Doppler-correction of variance into the band. The strongest corrections into the filter band took place in December 1985, February 1987, December 1990 and February 1991 (months 6, 11, 21 and 23). Other months exhibited smaller corrections or corrections out of the filter band (December 83, January and February 1984, January 1988, i.e., months $0,1,2,16)$.

The western Atlantic area mainly experienced corrections out of the filter band due to the high background wind speed in that region (Fig. 8). The negative corrections were mainly concentrated in the seasons 89/ 90-91/92 (months 18-26). These seasons did not actually exhibit exceptionally high monthly mean background wind speeds but happened to have a considerable amount of variability close to the cut off frequency.

\subsection{Daily variability}

The daily time series can illuminate the origins of the monthly mean corrections. In this section the daily time series of the winter season, DJF 90/91 (months 21-23), a
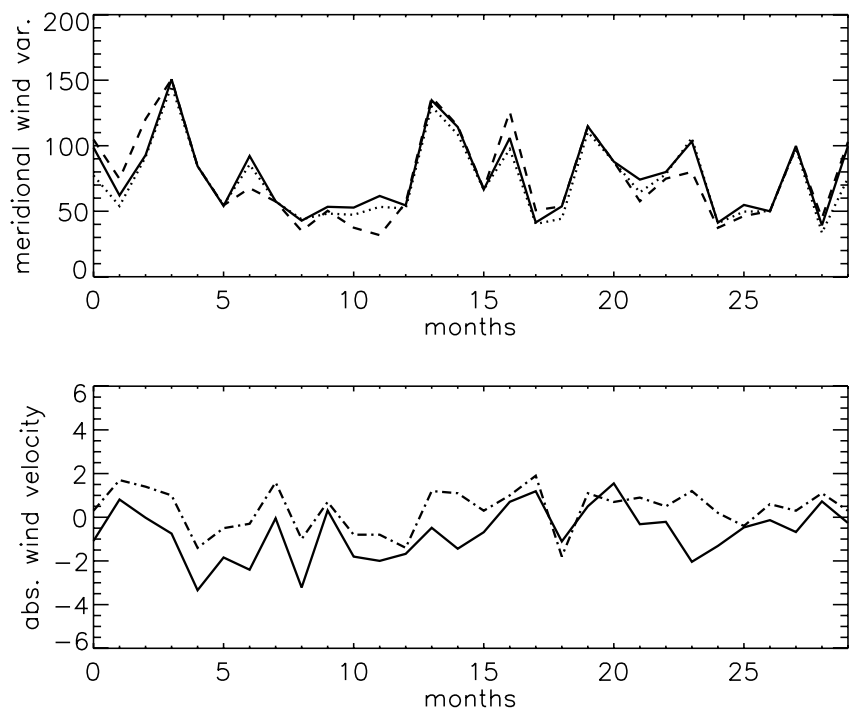

Fig. 7 As Fig. 6 but for the eastern Atlantic. The dash-dotted line in the lower panel shows the NAO index
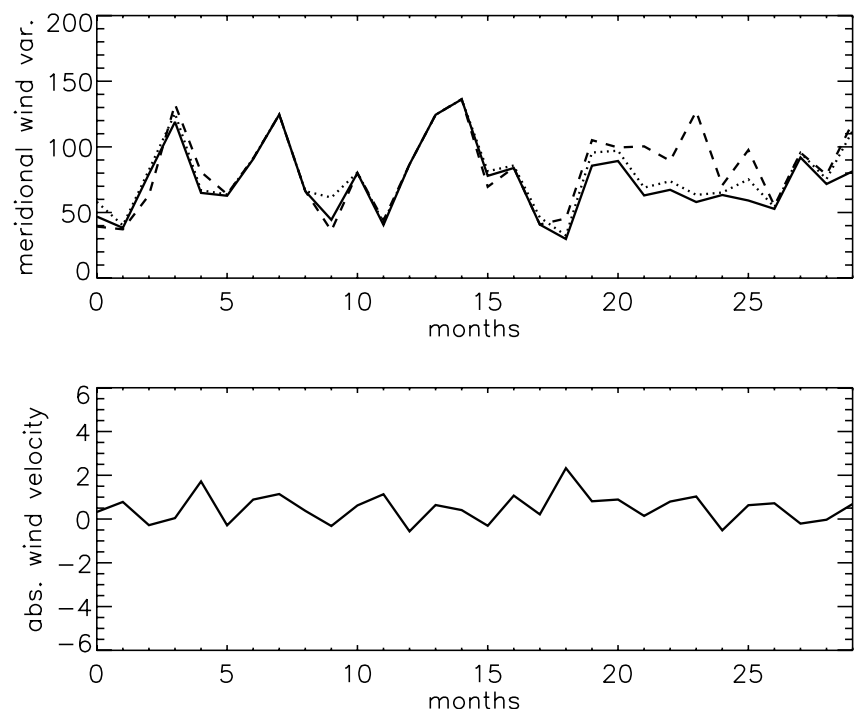

Fig. 8 As Fig. 6 but for the western Atlantic

season of strong Doppler correction in the European and eastern Atlantic area, is examined.

Figure 9 shows the daily time series of Lanczos filtered, EEOF-filtered and Doppler corrected variance of the meridional wind anomaly in the European area. Figure 10 shows the equivalent picture for the eastern Atlantic and Fig. 11 for the western Atlantic.

The agreement between the Lanczos filter and the EEOF filter in the daily time series is often rather poor.
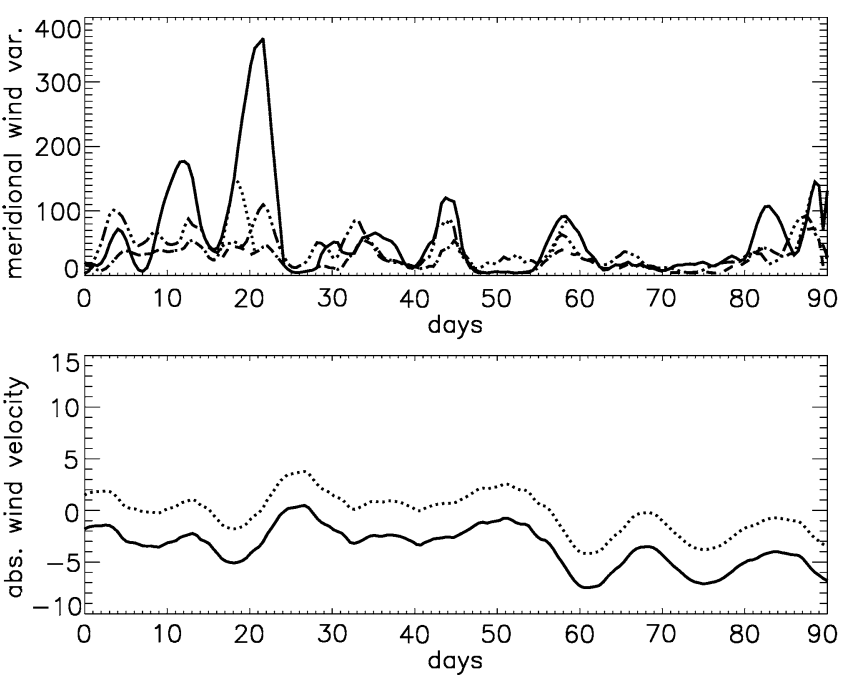

Fig. 9 Daily time series of the meridional wind variance (units $\mathrm{m}^{2} \mathrm{~s}^{-2}$ ) over Europe for the winter 90/91. The dashed line indicates the uncorrected time series, the solid line the Doppler corrected time series when correcting towards a mean background wind speed of $16 \mathrm{~m} \mathrm{~s}^{-1}$, the dotted line the corrected time series when correcting towards the regional and time averaged wind speed and the dashed-dotted line the Lanczos filtered time series. The lower plot shows the deviation of the background wind speed in $70 \mathrm{kPa}$ (units $\mathrm{m} \mathrm{s}^{-1}$ ) from $16 \mathrm{~m} \mathrm{~s}^{-1}$ (solid) and from the time averaged wind speed for that area (dotted) 

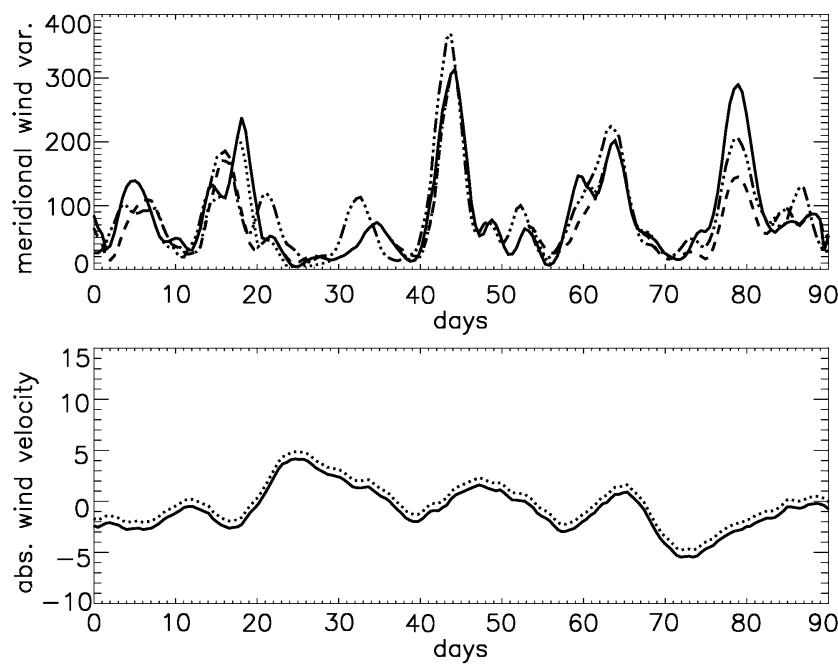

Fig. 10 As in Fig. 9 but for the eastern Atlantic

Very often, when a Doppler correction is applied, the Lanczos filtered time series lies in between the corrected and the uncorrected time series. Such behaviour is a consequence of the difference in the response functions of the filters. Unless the large scale flow speed at the time of correction is unusually low, the need for a Doppler correction generally implies that there is variability at frequencies very close to the cut off frequency of the filter band. Since the response of the Lanczos filter does not cut off sharply at the ends of the filter band but smoothly decreases to zero, the Lanczos filtered data will always include a proportion of the variability at frequencies only slightly longer than 6 days. This is exactly the variability that is corrected into the storm track measure at times of low wind speeds.

Again, the Doppler correction is not equally distributed over all those times when the background wind speed is low, but is concentrated in a few major events. The major Doppler correction into the storm track band
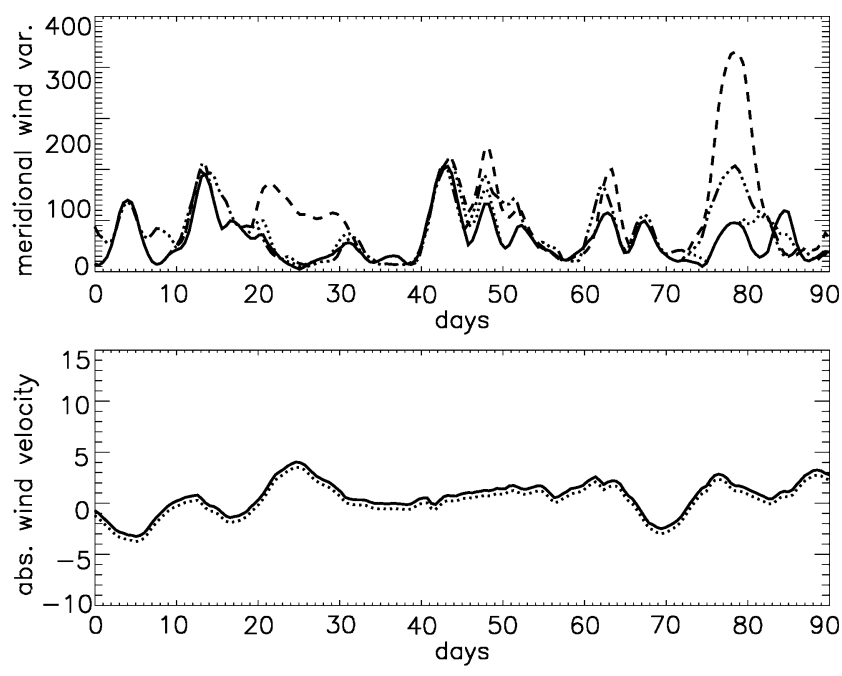

Fig. 11 As in Fig. 9 but for the western Atlantic in the European area happens around day 20, and to a lesser extent around day 11 and around day 82. The corrections at day 20 and day 82 will be examined more fully below.

Around day 20, the Hovmoeller diagram of the unfiltered wind (Fig. 1a) shows a very strong disturbance moving into the European area and crossing Europe in the following days. After the application of a Lanczos filter, this disturbance is partly contained in the 2-6 day band (Fig. 1b) and partly in the 6-10 day band (Fig. 1c). The variability in the 6-10 day band links separate maxima in the 2-6 day band. In the unfiltered Hovmoeller diagram and in the 10 day high pass filtered field (Fig. 3) these maxima are seen to be associated with the same disturbance. It is this variance in the 6-10 day band located between the high pass maxima that is Doppler-corrected into the storm track measure (Figs. 9, 3). The variability in the 6-10 day band associated with this disturbance was already relatively strong in the eastern Atlantic around day 18 (Fig. 1c). At this time, the background wind speed in the eastern Atlantic area was quite low, resulting in a relatively strong Doppler correction around day 18 (Fig. 10). The variability in the 2-6 day field in the western Atlantic area showed a peak around day 14 which was connected with the disturbance later found in the eastern Atlantic area and over Europe. At the same time there is very little variance in the 6-10 day field. Hence, Doppler correction did not have a significant effect in the western Atlantic area at that time (Fig. 11).

Around day 82, a moderate Doppler correction event occurs in the European area. Variability in the 2-6 day Hovmoeller diagram (Fig. 1b) was quite low whereas in the 6-10 day field, variability was moderately strong. At the time, the large scale wind speed was about average for the European area but was quite low compared with the storm track areas. Thus Doppler correction estimated an increased storm track activity (Fig. 9). The peak in storm track activity at around day 79 in the eastern Atlantic area was apparently connected with the same disturbance. At that time the disturbance was much stronger in the 2-6 day band in the eastern Atlantic than in the European area. But at the same time, significant variability was still contained in the 6 10 day band. This was shifted into the storm track band because of the relatively low background wind speed. This correction leads to large storm track activity in the Doppler-corrected time series (Fig. 10). As in the eastern Atlantic, in the western Atlantic the disturbance around day 78 was reasonably strong in the $2-6$ day band and a reasonably large fraction of the variability was also passed into the 6-10 day band. But since the background wind speed was high, the correction shifted variance out of the band (Fig. 11). Consequently, the variability due to this disturbance at day 78 was excluded.

Any storm track measure that relies on the phase speed to discriminate between baroclinic and lower frequency disturbances will include only part of the life 
cycle of a disturbance in the storm track measure at times and exclude the rest. The Doppler-corrected storm track measure has only the advantage of suppressing any bias due to the background flow. The exclusion of part of the life cycle of the cyclone could have been avoided by choosing a different wind speed towards which the scheme is correcting or by increasing the frequency range of the filter band. Such tuning would be unsatisfactory since it would only work for particular disturbances of particular frequency.

\section{Correcting for temporal variability only}

In this section, the impact of the Doppler correction on the variability of the storm track measure will be analyzed in the case when only the temporal variability of the background wind speed is corrected for. The wind speed was averaged in the analyzed area (e.g. Europe) for all ten seasons (DJF). The resulting wind speed anomalies (e.g. the time anomaly from the mean European wind speed) were used for the Doppler correction.

Figure 6 also shows the storm track activity over Europe after correcting for only the temporal variability of the large scale wind speed. As expected, Doppler correction had a much more subtle effect on the time series of the meridional wind variance than when correcting for both the temporal and spatial variability. In the winter $90 / 91$, the estimate of the monthly mean storm track activity changed little when correcting only for the temporal variability (Fig. 9). None of the major Doppler-correction events, when correcting for the spatial variability of the background flow as well, are still occurring.

When correcting the storm track measure only for the temporal variability of the large scale wind speed in the eastern Atlantic area (Fig. 7), the monthly mean corrections were bigger than in the European area. In the season 1983/84, in January 1989 and in February 1993 (months $0-2,16,29$ ), storm track activity was reduced by about $25 \%$. In December 1985, February 1987 and February 1991 (months 6, 11, 23), the Doppler correction resulted in a substantial increase in storm track activity relative to the uncorrected measure. In the season 1990/91, the two Doppler-corrected daily time series were almost identical (Fig. 10), so that monthly estimates for the storm track variability were very similar.

When correcting for the temporal variability of the large scale wind speed only in the western Atlantic area, the monthly mean time series (Fig. 8) was very similar to the time series when correcting for both temporal and spatial variability. The monthly mean corrections were slightly smaller because the average flow speed in that area was slightly higher than the $16 \mathrm{~m} \mathrm{~s}^{-1}$ (the value towards which the Doppler correction was performed in Sect. 5). In the season 1990/91, the two Doppler corrected time series were fairly similar in the western Atlantic. Differences between the two time series in February 1991 nearly cancelled out, so that the monthly mean Doppler corrected estimates of storm track activity were fairly similar.

\subsection{Connection with NAO}

It is interesting to consider how Doppler correction affects the correlation between storm track intensity and NAO index mentioned in the introduction. Together with the wind speed anomaly, the NAO index is plotted in Fig. 7. The two time series are significantly correlated, with correlation coefficient $r=0.703$. The corrections which lead to a decrease in storm track activity occur at times of a high NAO index (and therefore high wind speeds) whereas those corrections which enhance the storm track activity are applied at times of a low NAOindex. An exception is February 1991 (month 23), when the NAO-index and the estimated wind speed anomaly disagreed strongly. At other times of relatively high index, such as January 1986, December 1986 and February 1990 (months 7, 9, 20), or low index, such as January 1985 and December 1987 (months 4, 12), Doppler correction was very small or zero. The connection between storm track intensity and wind speed, which has previously been noted by Lau (1988), can be illustrated by averaging the meridional wind variance over the upper quartile of months with extremely strong background flow and the lower quartile of months with weak background flow. Using the EEOF filtered, but not Doppler corrected, time series of the meridional wind the meridional wind variance was about 1.4 times larger in the months of high large scale wind speed than in the months of low large scale wind speed (Table 1). However, when the Doppler-corrected storm track measure was averaged over the same months, the relationship disappeared and the storm track intensity in the two quartiles was nearly exactly the same. Even though the analysis consists only of ten winter seasons, the study nevertheless indicates that the effect of Doppler correction could very well be significant and should be taken into consideration when talking about the connection of large scale flow anomalies and storm track intensity.

\section{Discussion}

This paper discusses the interpretation of a storm track measure, based on filtered variances or co-variances,

Table 1 Variance of the meridional wind anomaly at $30 \mathrm{kPa}$ in the uncorrected and Doppler corrected storm track measure. Monthly mean variances were averaged over seven months exhibiting highest flow speeds at $70 \mathrm{kPa}$ (upper quartile) and 7 months exhibiting lowest flow speeds (lower quartile)

\begin{tabular}{lll}
\hline & Uncorrected & Doppler corrected \\
\hline Upper quartile flow speed & 78.4 & 67.4 \\
Lower quartile flow speed & 55.8 & 67.9 \\
\hline
\end{tabular}


such as used by Blackmon (1976), its connection with the variability of the background flow and the aptness of a Doppler correction of such a measure. The Doppler corrected measure is an accurate estimate if the scale of the disturbances is independent of the background wind speed. The uncorrected measure is accurate if the change in the background flow is exactly compensated for by a change in the scale of the disturbance which in turn modifies the intrinsic phase speed of the disturbance. When analyzing the wavelength of disturbances in observed data, a slight but statistically insignificant bias in the scale of the disturbances with the large scale wind speed was found. This bias was much stronger when using filtered data. This suggests that the influence of the variability of the background flow on the measure may be partly compensated for by a change of the intrinsic phase speed of the disturbances but that a full compensation is certainly not happening. This means that both corrected and uncorrected measures give limiting estimates of storm track intensity.

A simple scheme for Doppler correction was described. The resulting Doppler corrected storm track measure, as well as its uncorrected version, rely on the decomposition of the meridional wind anomaly into space-time patterns using an EEOF/MSSA analysis. The space-time patterns described relatively regular patterns for which the scale and period could be estimated, permitting the construction of a filtered time series. The uncorrected storm track measure based on the EEOF/MSSA filter agreed reasonably well with the Lanczos filtered measure. For the Doppler correction, an estimate of the strength of the background flow and an estimate of the scale and period of the disturbance together led to a corrected intrinsic frequency of the disturbance. From this, a Doppler corrected storm track measure was constructed.

The Doppler correction changes our view of the intensity and extent of the time mean storm track to some degree. The maximum intensity of the storm track defined by a Doppler corrected measure is slightly downstream of the maximum intensity defined by the uncorrected version. The largest corrections occur at the end of the storm tracks, extending the storm tracks further to the east and partly filling in the storm track gap over North America. This indicates that the baroclinic disturbances are travelling further into the continent before decaying than the uncorrected storm track measure would suggest. This is certainly not in conflict with observational studies (e.g. Petterssen 1956) in which storms can be seen travelling over the whole American continent before re intensifying in the baroclinic areas at the east coast. It also agrees well with a study by Anderson et al. (2003) (see their Fig. 1d, e) who, using feature tracking, found that using as a prefilter a 20 day high pass instead of a 2-6 day bandpass filter resulted in an increased track density and deeper cyclones and therefore in an more intense storm track over the continent compared to over the oceans. Their storm track therefore extends further into the east, an area of lower mean wind speeds, when allowing lower frequency variability to be identified as baroclinic disturbances as well, consistent with our results in Sect. 5. A mere widening of the filter band on the other hand does not change the pattern of the storm track but results only in an increased amplitude (Trenberth 1981, 1991) so that Doppler correction is still of importance even when widening the filter band.

The monthly mean time series of the intensity of the storm track was significantly changed by the Doppler correction at times. In some months, the change of the monthly mean intensity of the storm track was due essentially to a single disturbance that was corrected into the storm track measure. This fact is not a reason for concern. Even the traditional Eulerian variance maps are actually built up from a fairly small number of disturbances (see Fig. 1b), while nevertheless remaining remarkably stable from month to month. Doppler correction can change the original estimate of monthly mean storm track activity by a factor of as much as two in certain months, while in other months, it may hardly be changed.

Without Doppler correction, large storm track intensity in the East Atlantic is correlated with an increased large scale wind speed and therefore with the NAO. Such a correlation of the strength of the storm track either with the teleconnection patterns or merely with the flow speed has been documented in several other studies such as Lau (1988). Surprisingly, this correlation completely vanishes when Doppler correction is applied to the storm track measure. As discussed in Sect. 3, this estimate is a limiting case. So the true correlation may not be zero but should be significantly reduced. Since the Eastern Atlantic is not a strongly baroclinic area, our $70 \mathrm{kPa}$ large scale flow is to a good approximation the barotropic flow component. Given the potential importance of this result it would be important to reinvestigate this connection using more than ten winters of data. The ERA40 dataset would afford such a time series.

In general, there are significant limitations to the Doppler corrected storm track measure defined in this study.

1. The measure is defined only for large longitude and latitude sections and therefore cannot give the same spatially detailed information as the usual storm track measures.

2. The separation of the flow into eddies and larger scales is relatively difficult. This separation is attempted by the use of two non overlapping time filters and by spatial averaging of the low pass filtered wind speed.

3. The estimate of the background flow may be problematic at times of strong variations of the baroclinicity, such as occur towards the start of the storm track or during events such as blocking, when there are strong undulations of the low frequency wave patterns. In such situations, the method can 
overcorrect the storm track if the main baroclinic wave guide lies partly outside the analyzed latitudinal belt. On the other hand, our background wind speed seems to be a good approximation to the barotropic wind speed so that this problem is only of limited significance (Appendix 1).

4. The use of only the first few EEOF-patterns, those that exhibit relatively regular space and time variability, for the construction of the storm track measure generally leads to a lower estimate for the variability of the 2-6 day frequency band than the common Lanczos filtered storm track measure. The filtered field nevertheless exhibits very similar structures to the Lanczos filtered field. Smaller scale variability and variability that did not resemble a propagating wave in the Hovmoeller diagram was excluded from the storm track measure.

Despite these difficulties, Doppler correction can provide valuable additional information about the strength of the storm track, and can lead to an estimate of the error of an uncorrected measure. Doppler correction is likely to be particularly important when comparing the storm tracks in model simulations in which the large scale flow fields are quite different. Such a comparison is often attempted when analyzing the sensitivity of the storm track with simple GCMs (e.g. Lunkeit et al. 1998), in paleoclimate studies (e.g. Kageyama et al. 1999; Dong and Valdes 1998) or in climate change studies (e.g. Hall et al. 1994; Ulbrich and Christoph 1999).

Acknowledgements We are grateful to the Natural Environment Research Council who funded this work through grant number GR3/11298. The paper was much improved by the comments of three anonymous reviewers, to whom we extend our thanks.

\section{Appendix 1}

\subsection{Background flow speed}

The phase speed of baroclinically unstable disturbances can be described in terms of the 'steering level' or 'critical level of the flow'. In Eady's model (Eady 1949) the steering level is at the mid height whereas in Charney's model (Charney 1947) the steering level is proportional to the slope of the zonal mean isentropes and turns out to be very close to the Eady steering level for typical mid-latitude conditions. Using a jet similar to the North Atlantic jet, the steering level at the centre of the jet core still is approximately at $70 \mathrm{kPa}$ (James 1995, Fig. 5.24a). These considerations apply to the growth phase of small amplitude eddies. For mature or decaying eddies linear theory is little use in predicting the steering level. However, synoptic experience (e.g. Carlson 1991) and storm tracking studies (Hoskins and Hodges 2002) suggest that mid latitude cyclones move at about the speed of the $70 \mathrm{kPa}$ flow.
Even though $70 \mathrm{kPa}$ seems to be the relevant level for the movement of baroclinic cyclones in order for Doppler correction to be physically meaningful the wind speed anomaly $u^{\prime}$ must be to a good approximation the variability of the barotropic flow component (see Appendix 2). Figure 12 shows a winter time series of the $70 \mathrm{kPa}$ flow filtered with a 10 day low pass filter and averaged over $30^{\circ}$ latitude and $30^{\circ}$ longitude and an estimate of the barotropic flow component in the western Atlantic area, an area with relatively high baroclinicity. It seems that the variability of the background $u_{700}^{\prime}(x, t)$ is a reasonably good approximation of the variability of the barotropic wind component even in an area of relatively strong vertical wind shear such as the West Atlantic. This kind of low pass filtered and area averaged wind speed is termed the background wind anomaly or the large scale wind in this paper.

\section{Appendix 2}

\subsection{Normal mode theory}

Assuming $u^{\prime}(x, t)$ to be the variability of the barotropic component of the wind speed neither $k$ nor $\omega_{i}$ depend on $u^{\prime}$ according to Eady (1949) or Charney (1947). On the other hand $u^{\prime}(x, t)$ might be correlated with the variability in the vertical wind shear. According to Eady's theory of baroclinic instability (Eady 1949) the wavelengths of the fastest growing modes, $k$, do not depend on the vertical wind shear and the intrinsic phase velocity is zero at half level. According to Charney's theory of baroclinic instability (Charney 1947) $k^{-1}$ is proportional to the vertical wind shear

$k^{-1}=1.26 \frac{f_{0}}{\beta N} \frac{\delta u}{\delta z}$

and therefore (under the assumption of $u^{\prime}$ being correlated with the vertical wind shear) to $u^{\prime}$. As the vertical wind shear increases, the scale of the disturbance decreases and the phase velocity increases. Therefore the change in $k$ would lead to a partial compensation of the change in the background flow speed so that Doppler

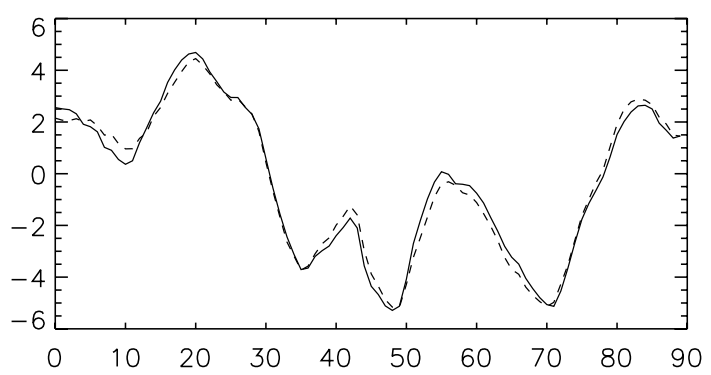

Fig. 12 Anomaly of the barotropic flow component (solid line) and the flow at $70 \mathrm{kPa}$ (dashed line) during one winter month in the West Atlantic area 
correction would lead to an overcorrection of the effect of variable strength of the background flow.

Disturbances predicted by the Eady or Charney models are assumed to grow linearly from infinitesimally small disturbances. It is very questionable if the properties of the disturbances observed in nature or simulated by global circulation models can be easily predicted by any of those theories (see also Lee and Mak 1996 or discussion in Chang et al. 2002). Observed disturbances rarely grow from infinitesimally small perturbations but rather from finite amplitude initial disturbances. Such disturbances may be orographically generated or may be remnants from an upstream storm track. The size of initial disturbances will probably have a greater influence on the scale of the developed eddies than the scale of the fastest growing linear mode. Baroclinic instability theory can therefore give no clear answer about the dependency of the wavelength on the wind speed $u_{700}$ when assuming the variability of $u_{700}$ to be associated with the variability of the vertical wind shear. In the case of the variability of $u_{700}$ being due to the variability of the barotropic wind component Doppler correction would be eliminating a systematic error of the storm track measure.

Another highly idealized theory is a barotropic storm track which for small amplitude waves can be approximated by WKB theory (Lee 1995; Swanson et al. 1997). It assumes a scale separation between a barotropic background flow and a small amplitude wave and predicts a change of $\mathrm{k}$ with the flow so that wave activity is conserved. Assuming small amplitude waves and scale separation and that the dynamics of barotropic storm tracks dominate the problem, Doppler correction would not be necessary. Both Lee and Swanson et al. find that barotropic effects may be of importance for the localization of storm tracks though they disagree on the strength of the effect (Swanson et al. 1997; Chang et al. 2002). Swanson et al. infer that nonlinearities modulate barotropic eddy activity more strongly than linear processes. Comparing linear barotropic model experiments and observational data Lee (2000) concludes that the storm tracks are localized due to different processes in observations and in the model, and that barotropic dynamics are more relevant for lower latitude storm tracks such as the North African and Middle Eastern storm track than for the midlatitude Atlantic and Pacific storm tracks.

\section{References}

Anderson D, Hodges KI, Hoskins BJ (2003) Sensitivity of featurebased analysis methods of storm tracks to the form of background field removal. Mon Weather Rev 131:565-573

Blackmon ML (1976) A climatological spectral study of the $500 \mathrm{mb}$ geopotential height of the northern hemisphere. J Atmos Sci 33:1607-1623

Blackmon ML, Wallace JM, Lau N-C, Mullen SL (1977) An observational study of the northern hemisphere wintertime circulation. J Atmos Sci 34:1040-1053
Carlson TN (1991) Mid-latitude weather systems. Harper Collins, London, $507 \mathrm{pp}$

Chang IKM, Lee S, Swanson KL (2002) Storm track dynamics. J Clim 15:2163-2183

Charney JG (1947) The dynamics of long waves in a baroclinic westerly current. J Meteor 4:135-162

Dong B, Valdes PJ (1998) Siumulations of the last glacial maximum climates using a general circulation model: prescribed versus computed sea surface temperatures. Clim Dyn 14:571591

Duchon CE (1979) Lanczos filtering in one and two dimensions. J App Met 18:1016-1022

Eady ET (1949) Long waves and cyclone waves. Tellus 1:33-52

Fraedrich K, Bantzer C, Burkhardt U (1993) Winter climate anomalies in Europe and their associated circulation at 500 hPa. Clim Dyn 8:161-175

Gibson JK, Kallberg P, Uppsala S, Hernandez A, Nomura A, Serrano E (1997) ECMWF Reanalysis, Project Report Series. 1. ERA Description

Gill AE (1982) Atmosphere-ocean dynamics, international geophysics series. Academic, New York, 662pp

Hall NMJ, Hoskins BJ, Valdes PJ, Senior CA (1994) Storm tracks in a high-resolution GCM with doubled carbon dioxide. Quart J Roy Meteor Soc 120:1209-1230

Hoskins BJ, Hodges KI (2002) New perspectives on the northern hemisphere winter storm tracks. J Atmos Sci 59:10411061

Hoskins BJ, James IN, White GH (1983) The shape, propagation and mean-flow interaction of large-scale weather systems. J Atmos Sci 40:1595-1612

James IN (1995) Introduction to circulating atmospheres. Cambridge University Press, Cambridge, 422pp

Kageyama M, Valdes PJ, Ramstein G, Hewitt C, Wyputta U (1999) Northern hemisphere storm tracks in present day and last glacial maximum climate simulations: a comparison of the European PMIP models. J Clim 12:742-760

Lau N-C (1988) Variability of the observed midlatitude storm tracks in relation to low-frequency changes in the circulation pattern. J Atmos Sci 45:2718-2743

Lee S (1995) Localized storm tracks in the absence of local instability. J Atmos Sci 52:977-989

Lee S (2000) Barotropic effects on atmospheric storm tracks. J Atmos Sci 57:1420-1435

Lee W-J, Mak M (1996) The role of orography in the dynamics of storm tracks. J Atmos Sci 53:1737-1750

Lunkeit F, Fraedrich K, Bauer SE (1998) Storm tracks in a warmer climate: sensitivity studies with a simplified global circulation model. Clim Dyn 14:813-826

Metz W (1989) Low frequency anomalies of atmospheric flow and the effects of cyclone-scale eddies: a canonical correlation analysis. J Atmos Sci 46:1027-1041

Mullen N-C (1986) Transient eddy forcing of blocking flows. J Atmos Sci 44:3-22

Nakamura H (1989) Midwinter suppression of baroclinic wave activity in the Pacific. J Atmos Sci 49:1629-1642

Petterssen S (1956) Weather analysis and forecasting, vol 1. McGraw-Hill Book Company, Inc.,New York, 428pp

Plaut G, Vautard R (1993) Spells of low-frequency oscillations and weather regimes in the northern hemisphere. J Atmos Sci $51: 210-236$

Simmons AJ, Hoskins BJ (1978) The life cycles of some nonlinear baroclinic waves. J Atmos Sci 35:1454-1477

Swanson KL, Kushner PJ, Held IM (1997) Dynamics of barotropic storm tracks. J Atmos Sci 54:791-810

Trenberth KE (1981) Observed southern hemisphere eddy statistics at $500 \mathrm{mb}$ : frequency and spatial dependence. J Atmos Sci 38:2585-2605

Trenberth KE (1991) Storm tracks in the southern hemisphere. J Atmos Sci 48:2159-2178

Ulbrich U, Christoph M (1999) A shift of the NAO and increasing storm track activity over Europe due to anthropogenic greenhouse gas forcing. Clim Dyn 15:551-559 
Vautard R (1995) Patterns in time: SSA and MSSA. Analysis of climate variability. In: von Storch H, Navarra A (eds) Applications of statistical techniques. Springer, Berlin Heidelberg New York, pp 259-279

Wallace JM, Gutzler DS (1981) Teleconnections in the geopotential height field during the northern hemisphere winter. Mon Wea Rev 109:784-812
Wallace JM, Lim G-H, Blackmon ML (1988) Relationship between cyclone tracks, anticyclone tracks and baroclinic waveguides. J Atmos Sci 45:439-462

Weare BC, Nasstrom JS (1982) Examples of extended empirical orthogonal function analyses. Mon Wea Rev 110:481-485

Wilks DS (1995) Statistical methods in the atmospheric sciences. Academic, New York, 464pp 